\title{
Impact Noise of Non-homogeneous Floors: Analysis of Different Input Parameters for Computational Modeling Predictions
}

\author{
Maria Fernanda de Oliveira ${ }^{1}$ and Jorge Viçoso Patricio ${ }^{2}$ \\ 1. Technological Institute onCivil Construction - itt Performance, Unisinos University, São Leopoldo 93022-750, Brazil; \\ 2. National Laboratory for Civil Engineering-LNEC, Lisbon1700-066, Portugal
}

\begin{abstract}
Floor systems with non-homogeneous slabs have more complex means of propagation than homogeneous systems, with more variables to be considered in predictions by theoretical models. For those slabs, it is necessary to understand the differences of each material composing each subsystem, and the connection types between the elements of each one of this subsystem. Some floors integrating lightweight elements without structural purposes, are broadly used in several countries in precast slabs. The predictions based on computer modelling for building systems can be influenced by the input parameters related to connections between the elements of the floor system. In building structures, the analysis of radiation due to element vibrations may be represented by wave propagation relationships as a one-dimensional system, a two-dimensional system or a three-dimensional solid. In these floors, the modelling of the interaction between elements can be basically a face, a line or a point connection. In addition, the choice of the connection type can determine the vibration transmission amongst all the floor elements. This study focuses on the differences that can be obtained in the induced vibration response due to an impact source on non-homogeneous slabs. It also presents some examples of modelling options for several floor systems, considering input parameters for different connection types.
\end{abstract}

Key words: Non-homogeneous floors, vibrational response, finite element analysis.

\section{Introduction}

Vibration transmission estimates of buildings can provide relevant information for analysis of irradiation for different construction components of multi-story buildings. Several aspects are studied for the acoustic behavior analyses of different types of floors in order to propose efficient design solutions. The type of building structures is one of the first aspects to be analyzed, since wave propagation is different for heavy and lightweight components.

The type of connection between components of floor systems should represent the way they were built, but estimates by computer models connections must represent the form of vibration transmission which

Corresponding author: Maria Fernanda de Oliveira, Dr., associate professor; research fields: building acoustics, building performance and sound insulation. E-mail: mariaon@unisinos.br. occurs between attached components.

The input parameters for the prediction of vibrational response of floors can influence the results in modelled building systems. The element type assigned to represent the ribs is the main issue in modelling plate/beam systems, more specifically, the choice of the connections type [1]. In building structures, the analysis of radiation due to beam vibrations may be represented from the wave propagation relationships as a one-dimensional system, a two-dimensional system or a three-dimensional solid. Thus, the different forms of representation beam and plate systems of computer models can give results which do not represent the vibrational response in non-homogeneous floors [2].

The structural elements of buildings are geometrically formed from the combination of plates and beams, which are means for irradiating sound 
resulting from bending waves. Basically, the reduction of mobility of such elements is a fundamental solution for reducing the impact sound of floors. For infinite elements, the mobility flexure of plates and beams are inversely proportional to their thickness, but for finite elements, the peripheral boundary conditions strongly influence the mobility of these elements [3]. Because it is a structural element, whose main function is to create longer spans without intermediate supports, the options of mobility change for concrete slabs and hence the amplitude of the vibrational response, are in the dimensions of the cross sections of their elements.

The non-homogeneous floors are an appropriate solution from a structural point of view, however, the acoustic performance to be characterized from the range of options that combine slabs, plates and beams in composition [4]. Therefore, the flexural rigidity in the parallel direction to the concrete beams is determined by the geometry of those beams. In addition, if the blocks have a very low mass compared to concrete screed, it is possible to ignore it and use the ribbed slab model for the other beams and plate models [5].

Vibration irradiation of structures is directly related to the stiffness of their elements, for which there is great complexity in determining the vibration modes of plates when reinforced by beams. The rigidity provided by beams also serves to the cancellation of the compression regions and existing rarefaction on the plates surfaces, resulting from transverse displacements in orthogonal directions [6]. Furthermore, the structural reinforcement of beams changes the displacements plate distributions, which causes no cancellation regions close to these beams and produces an increased noise radiating plate in these regions. The magnitude of this irradiation depends on the effects of inertia and beams rigidity [6, 7].

This study focuses on the differences that can be obtained in the vibrational response due to an impact source exerted on non-homogeneous slabs. It also presents examples for modelling options for plate/beam systems with and without ceiling, considering different input connection types for 2D and 3D models.

\section{Modeling Parameters}

The validation of the model was made by a comparison with measurements in LNEC (National Laboratory for Civil Engineering, in Portugal), for which the frequency of the 8th slab modes used for impact sounds was compared with 2D and 3D models generated in Ansys ${ }^{\circledR} 16.2$ Workbench software. Two connections types between elements for each model have been tested: the connections to surfaces or FC (face connections) and LC (line connections).

The results indicate that the 2D model with face connections better expresses the vibrational response of the slab. On the other hand, the 2D model with line connections shows the greatest differences in the 8th mode (Fig. 1).

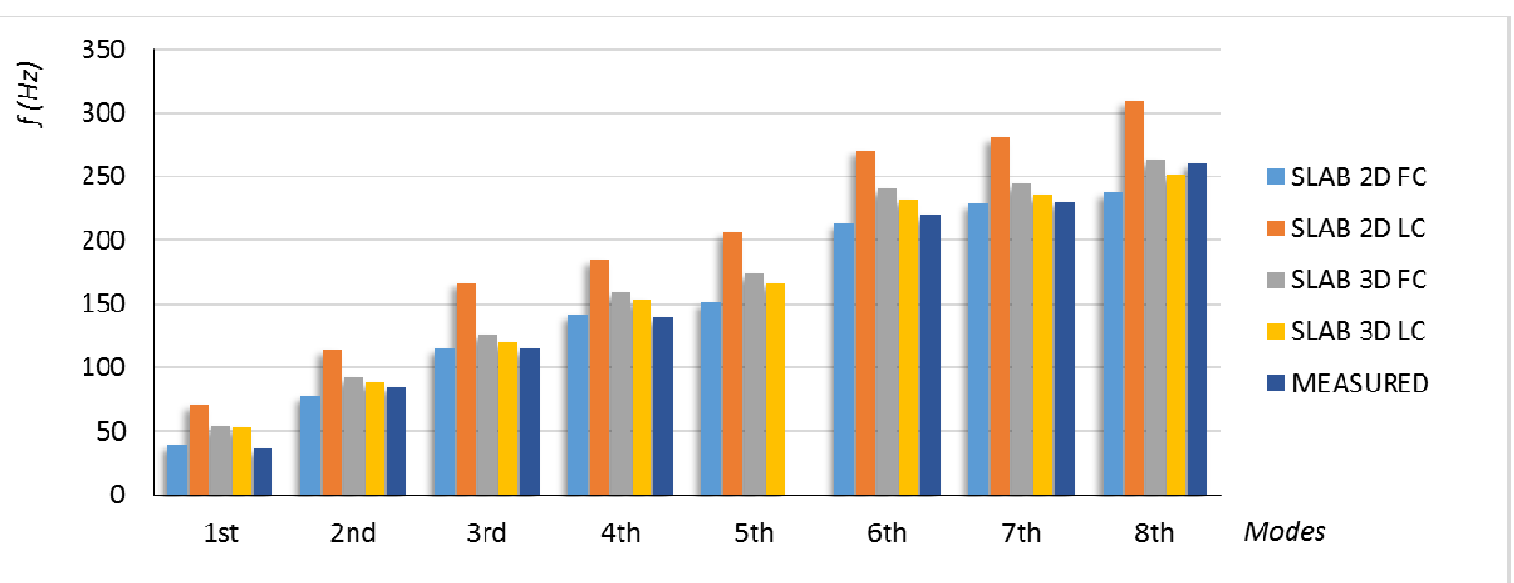

Fig. 1 Comparison between natural frequencies of the reference laboratory slab tests, and the 2D and 3D models. 
The choice of non-homogeneous slabs became from the main variables identified for the differences in vibrational responses in computer simulation models, and in the established relationships of similarities between the types of slabs used in buildings construction.

These variables are basically due to the connections between the three basic elements of a non-homogeneous concrete slab: beams, plate and the spaces between the beams.

The latest can or cannot be filled in because it is not a tensioned region and does not structurally contribute to the system. These spaces are usually filled in by lightweight blocks (hollow ceramic or concrete or EPS - expanded polystyrene) and in the case of slabs type beam/block, there is no rigid connection with the beams, existing instead narrow gaps between these elements.

For this study with non-homogeneous slabs, the followings were adopted: two slabs beam/block type with concrete beams and hollow concrete blocks, and solid EPS blocks; two ribbed concrete slabs with and without solid EPS blocks; and, a precast slab T-type.
For all models, it was considered a concrete screed with $5 \mathrm{~cm}$ thick.

For beam/block slabs type (BB and BB EPS) connections between beams and blocks were considered as dry, where input parameters were expressed as "unbonded". However, between concrete screed and other elements, the connections were considered as "bonded" (Fig. 2).

Ribbed slabs were also investigated from the point of view of influence of the spaces between the beams with (RC EPS) and without EPS blocks (RC); because this is one of the types currently present in Brazilian buildings constructions. Due to characteristics of the construction of this slab, concrete capping was considered as one element in relation to the beams (Fig. 3).

The T-type slab is formed by precast elements of which the module consists of a beam and the respective board, with a discontinuity between each module. Thus, the connection between the modules is affected by the top layer formed by the concrete screed (Fig. 4).

For the boundary conditions adopted in the models, it was assumed the same characteristics as used in the

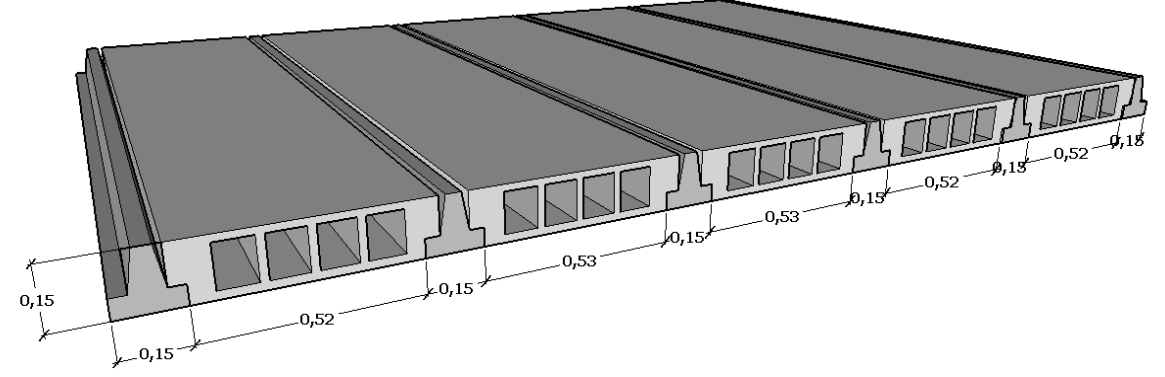

(a)

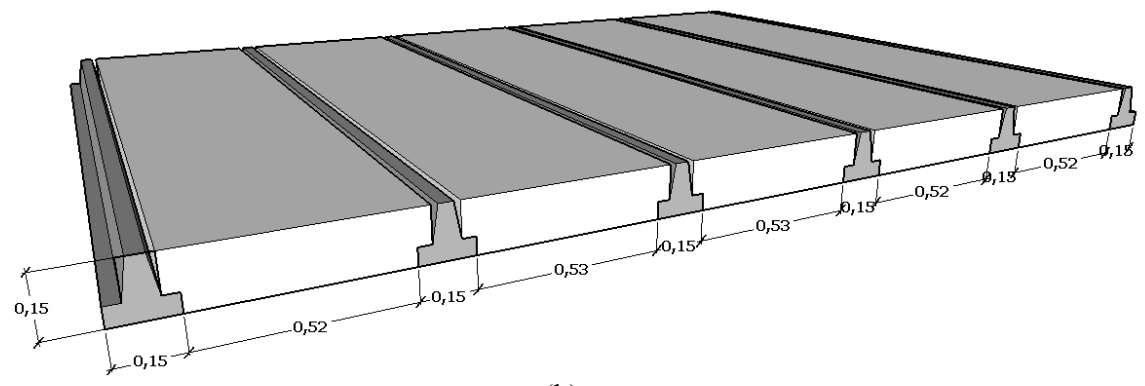

(b)

Fig. 2 Beam/block slab: (a) with concrete hollow blocks (BB); (b) with solid EPS block (BB EPS). 


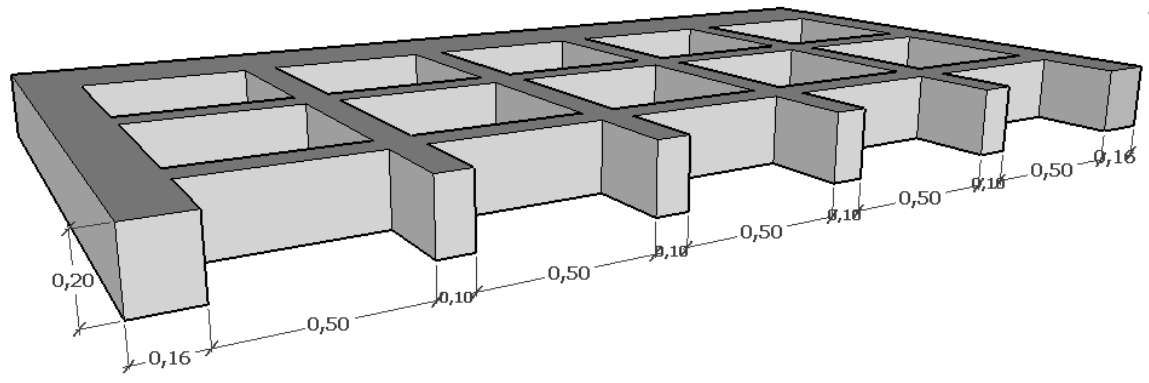

(a)

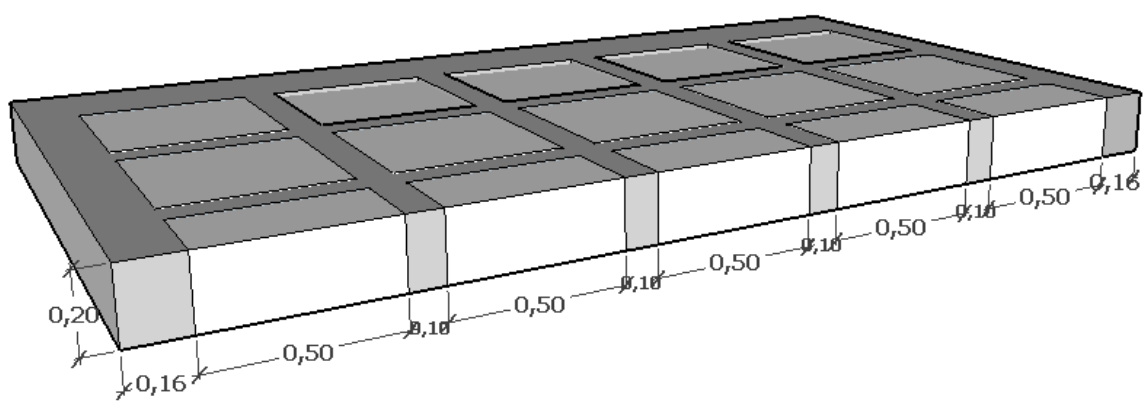

(b)

Fig. 3 Ribbed concrete slab: (a) without blocks (RC); (b) with solid EPS block (RC EPS).

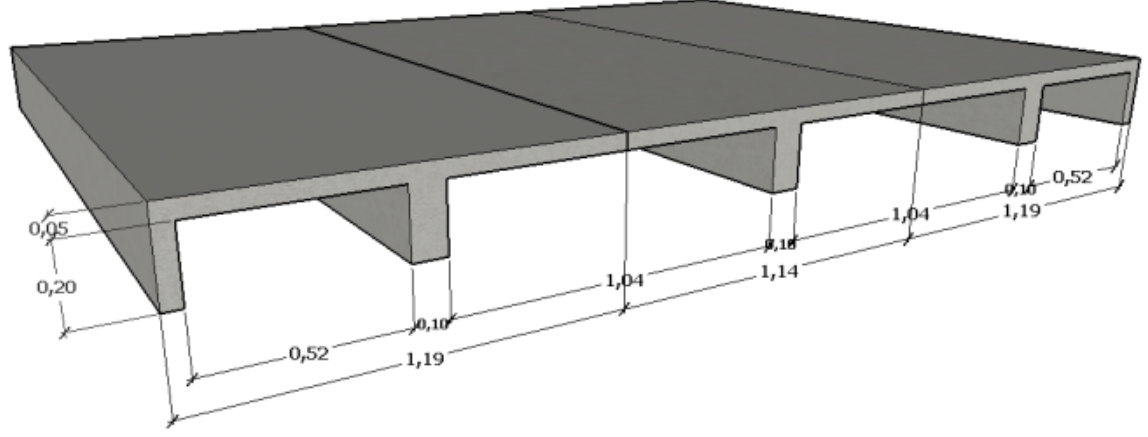

Fig. 4 T-type slab (TT).

initial model validation for the LNEC concrete slab (which is simply supported due to its contour resilient layer).

For comparative analysis, it was modeled two types of impact excitation force, for which the third octave bands variations aimed to represent a hard and an elastic covering.

The parameters used were based on another publication [8] which indicate that the decay of excitation force begins at the $500 \mathrm{~Hz}$ frequency band for an elastic coating, in comparison to other rigid coatings.

\section{Results}

Fig. 5 shows comparisons between the vibrational responses of slabs with a rigid covering, exhibiting different resonance peaks. The largest amplitude peaks occurred for the ribbed concrete slabs. T-type slab did not present a vibrational response so large compared to the others. Comparatively, the rigidity of the ribbed slab does not represent greater efficiency in reducing vibrations irradiation.

Comparatively, it can be pointed out that the largest irradiation efficiency occurs at the frequency band of 


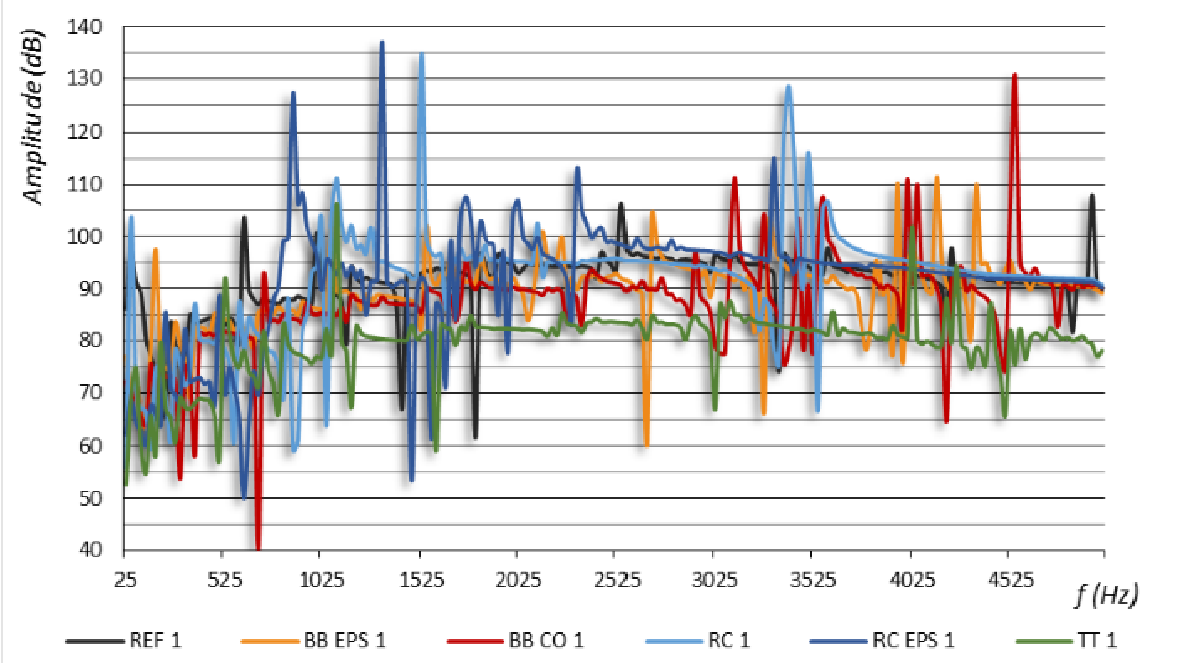

(a)

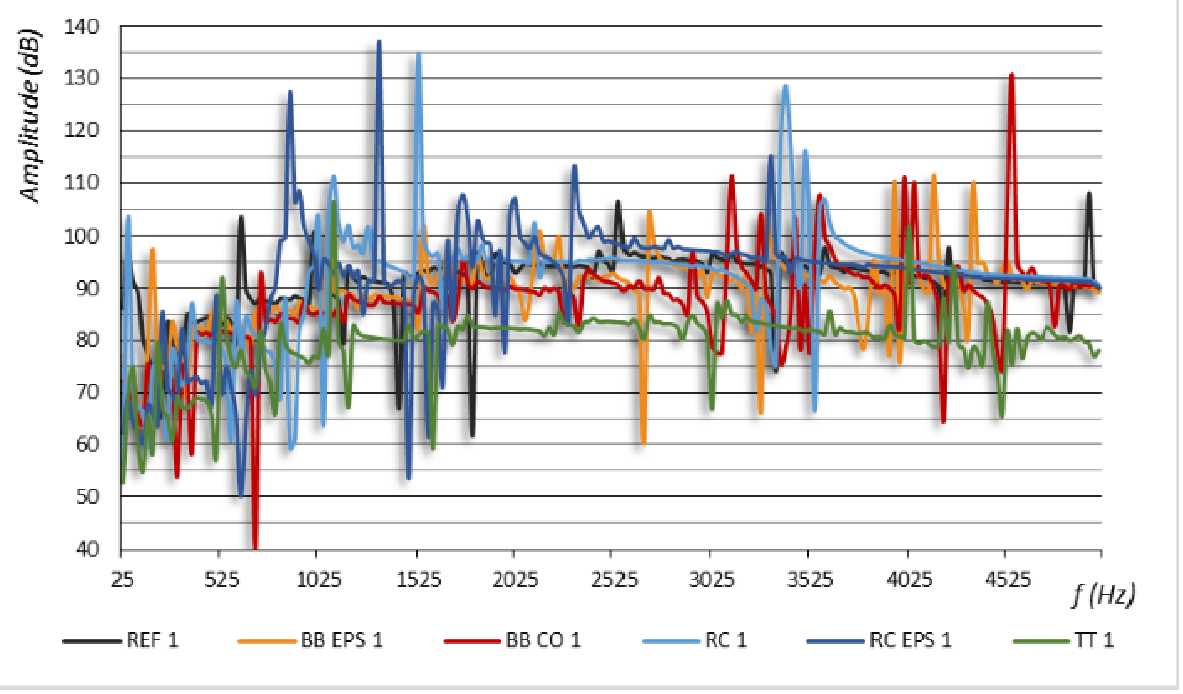

(b)

Fig. 5 Comparison of amplitude responses between slabs:(a) up to $5 \mathrm{kHz}$;(b) up to $600 \mathrm{~Hz}$.

$380 \mathrm{~Hz}$, which is the critical frequency of $5 \mathrm{~cm}$ thick of the concrete screed.

In Fig. 6, it can be seen the results of models simulations with rigid and elastic coverings, identified by the numbers 1 and 2, respectively. In all modeled slabs, there were reductions in the vibrational response in the mid and high frequencies without, however, changing the frequencies resonant peak. There was just a displacement in curves in relation to the decrease in amplitude results.

Ribbed concrete slabs can be performed with or without blocks in the space between the beams. The peaks of vibrational responses occurred at $1,525 \mathrm{~Hz}$
(Fig. 7b) for slab without EPS blocks and at 1,325 Hz for slab with EPS blocks (Fig. 7c). It can be pointed out that the presence of blocks slightly changed the beams mobility, where ribbed slab without blocks have clearly identified the major bending in the direction of greater span ( $\mathrm{xx}$ ’ direction).

In contrast, the EPS blocks may have an irradiation distribution effect also for the beams with lower span (yy' direction). This does not result in an improvement for vibrations reduction and, considering the tested examples, the resilient characteristics of the covering will be more relevant for the vibrational response of the floor system. For visualization of vibration response of 


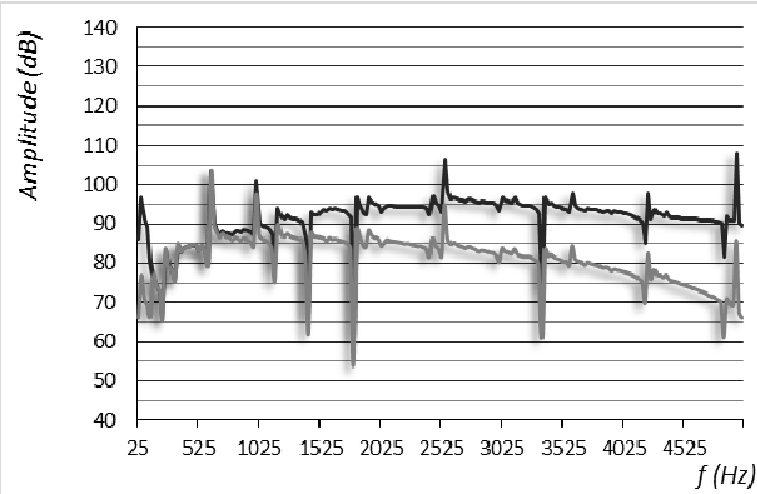

- REF 1

$\longrightarrow$ REF 2

(a)

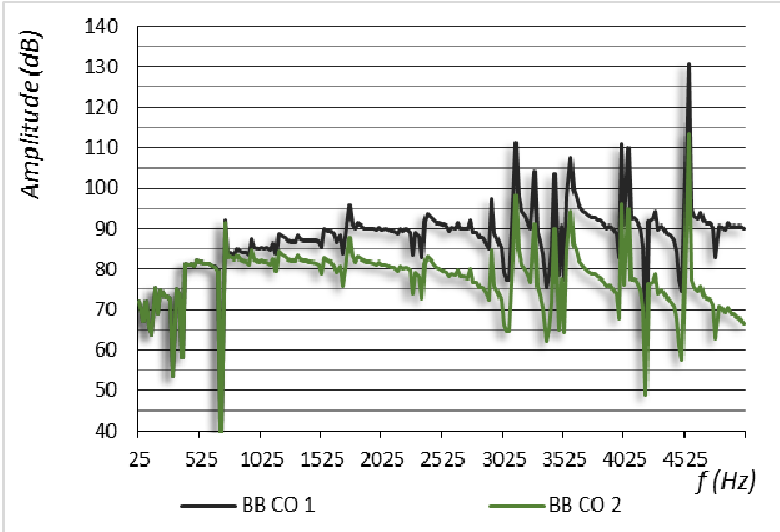

(c)

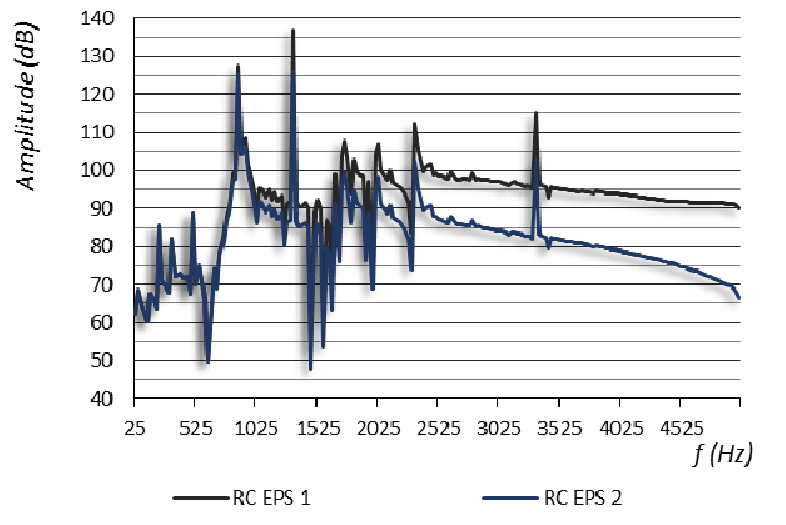

(e)

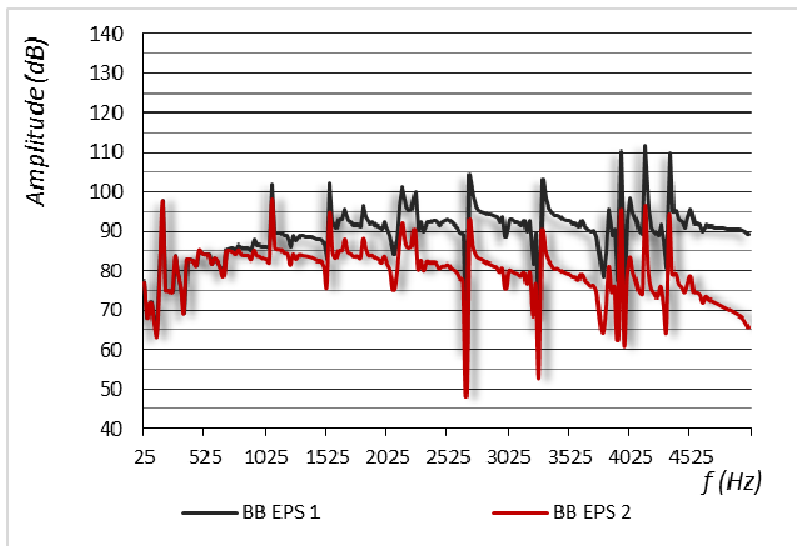

(b)

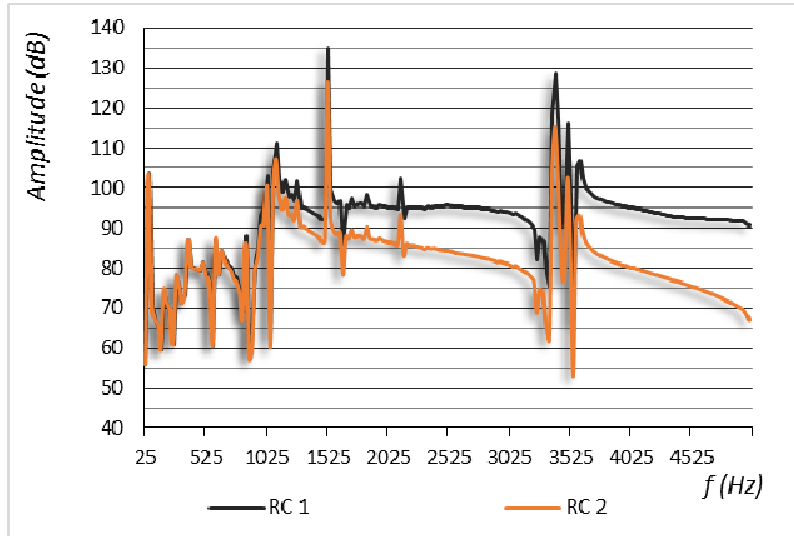

(d)

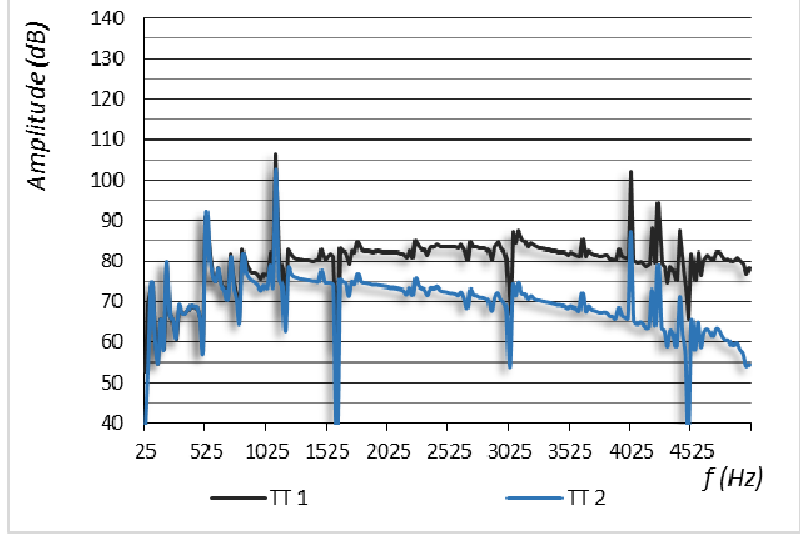

(f)

Fig. 6 Comparisons of amplitude responses for the slab with and without covering: (a) reference slab REF; (b) beam/block with EPS block BB EPS; (c) beam/block with concrete block BB CO; (d) ribbed concrete no blocks RC; (e) ribbed concrete with EPS blocks RC EPS; (f) T-type TT. 


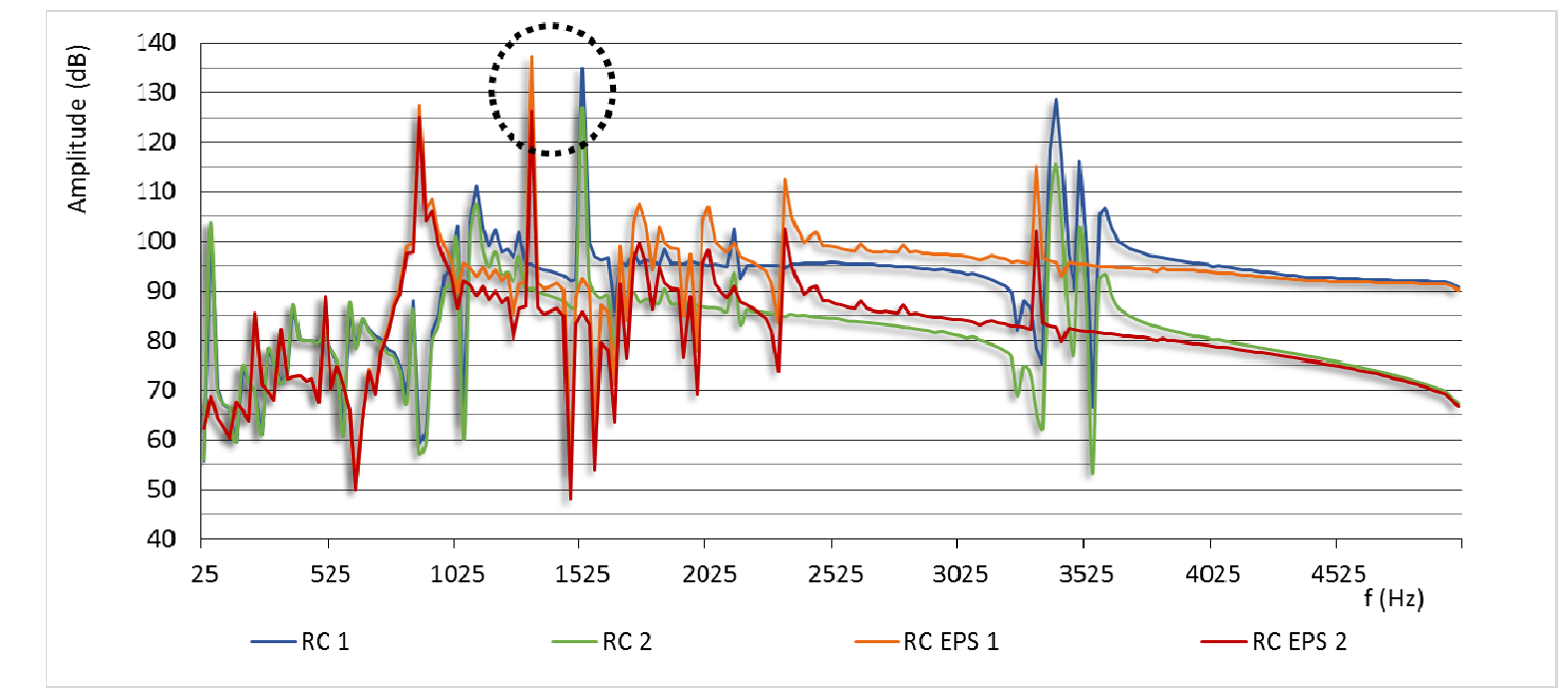

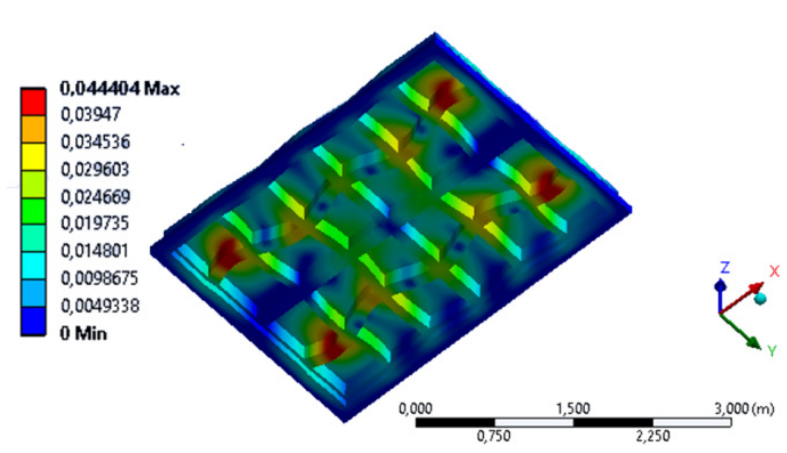

(b)

(a)

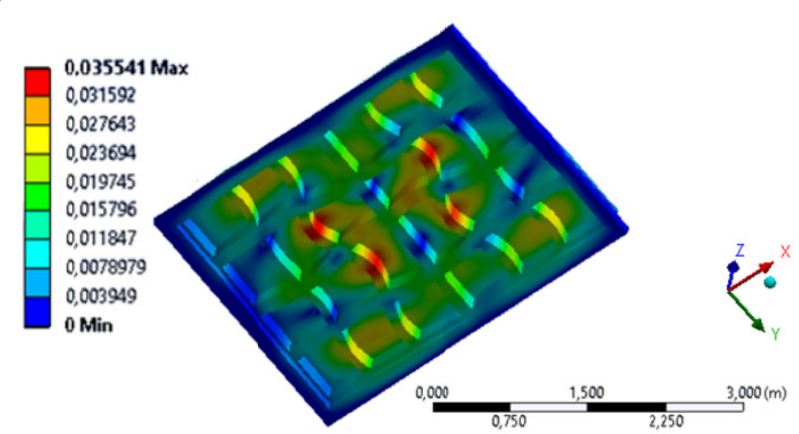

(c)

Fig. 7 Ribbed concrete slabs: (a) comparisons of amplitude responses; (b) mode of peak response for slab without block; (c) mode of peak response for slab with EPS blocks.

beams, the EPS blocks were hidden; only for graphic representation (but, they were considered in prediction models).

\section{Final Remarks}

This paper presents an investigation on the experimental and computer simulations related to characteristics of vibrational response of different non-homogeneous floors systems. The obtained results indicate that the resonance of the tested slabs is strongly influenced by rigidity provided both by the concrete screed and by the characteristics of the beams section.

Due to the geometry complexity and non-homogeneous characteristics of some slab types for large spans, estimating the vibrational response in this type of floors requires methods to consider their different built forms.
Briefly, in this comparative study of non-homogeneous floors, it can be pointed out that:

- concrete screed used on beam/block, ribbed and T-type slabs provides some sort of constraints that can allow a reduction of the mobility of the integrated elements;

- different solid elements formed by concrete can be represented as a single element;

- addition of elastic coverings can generate vibrational responses more similar to the expected behavior of real floors.

Studies with computer models are particularly suitable for comparative analysis in multi-parametric evaluations that can use the same model for different simulations types, because some characteristics defined in structural design can or cannot determine unfavorable conditions for the effectiveness of irradiation of vibrations. 


\section{Acknowledgments}

The authors thank the Brazilian National Council for Scientific and Technological Development (CNPq) for the Research Fellowship PDE (Process Number 201973/2015-4).

\section{References}

[1] Galbrun, L. 2010. "Vibration Transmission through Plate/Beam Structures Typical of Lightweight Buildings: Applicability and Limitations of Fundamental Theories." Appl. Acoust. $71 \quad$ (2010): $\quad 587-96$. doi:10.1016/j.apacoust.2010.01.009.

[2] Nunes, M. F. O., and Patrício, J. V. 2016. "Comparative Study of Different Modeling Parameters for Predicting Vibrations on Timber-Frame Floors Modeling Parameters." Presented at Congr. EuroRegio2016, 9th Iber. Congr. Acoust. 47th Spanish Congr. Acoust., SPA, Porto, 2016.
[3] Howard, C. Q., and Cazzolato, B. S. 2014. Acoustic Analyses Using Matlab ${ }^{\circledR}$ and Ansys ${ }^{\circledR}$. Boca Raton: Taylor $\&$ Francis.

[4] Patrício, J. V. 2001. "Can Beam-Block Floors Be Considered Homogeneous Panels Regarding Impact Sound Insulation?.” Build. Acoust. 8 (2001): 223-36. doi:10.1260/1351010011501920.

[5] Hopkins, C. 2012. Sound Insulation. Burlington: Elsevier Ltd.

[6] Fahy, F., and Walker, J. 2005. Advanced Applications in Acoustics, Noise and Vibration. New York: Taylor \& Francis.

[7] Peters, R. J., Smith, B. J., and Hollins, M. 2013. Acoustics and Noise Control. 3rd ed. New Jersey: Taylor \& Francis.

[8] Rabold, A., Buchschmid, M., Düster, A., Müller, G., and Rank, E. 2011. "Modelling the Excitation Force of a Standard Tapping Machine on Lightweight Floor Structures." Build. Acoust. 17 (2011): 175-97. doi:10.1260/1351-010X.17.3.175. 